\title{
Erratum: Spin dynamics of hot excitons in diluted magnetic semiconductors with spin-orbit interaction [Phys. Rev. B 100, 045306 (2019)]
}

F. Ungar, P. I. Tamborenea, and V. M. Axt

(Q) (Received 31 July 2019; published 12 August 2019)

DOI: 10.1103/PhysRevB.100.089901

In contrast to the expression in the original paper, the typical timescale of the spin-orbit interaction (SOI) should read $\tau_{\mathrm{SOI}}=$ $h\left(\alpha_{\mathrm{R}} \bar{K}\right)^{-1}$. Using the parameters of Fig. 1, we then find $\tau_{\mathrm{SOI}} \approx 12.4$ and $\tau_{\mathrm{SOI}} \approx 56.4$ ps for $\mathrm{Cd}_{1-x} \mathrm{Mn}_{x} \mathrm{Te}$ and $\mathrm{Zn}_{1-x} \mathrm{Mn}_{x} \mathrm{Se}$, respectively, which are a bit longer compared with the previously reported values. Apart from the change in estimated dephasing times, no results in the paper are affected. 